\title{
Vorwort der Präsidentin
}

Liebe DMV-Mitglieder,

im DMV-Server ist eine neue Rubrik „Stellenangebote" eingerichtet worden. Sie können dort Stellenangebote aus dem Universitätsbereich, der Wirtschaft und anderen Bereichen abfragen und andererseits auch selbst eingeben.

Über die viel beachtete internationale Mathematikstudie TIMSS (Third International Mathematics and Science Study) hat es Ende Juni eine Anhörung der Kultusministerkonferenz in Bonn gegeben, an der Herr Törner für die DMV teilgenommen hat. Die DMV-Stellungnahme, die dort als Tischvorlage ausgeteilt wurde, finden Sie im DMV-Server unter der Rubrik „Presseschau“.

Im Heft 1/97 der Mitteilungen haben die Herren Epple, Kneser und Speck über das DMV-Archiv in Freiburg berichtet und dabei auf das Fehlen einiger Aktenbestände hingewiesen. Diese Akten sind danach aufgefunden worden und befinden sich nun vollzählig im Archiv. Dank der Unterstützung durch die Volkswagen-Stiftung wird zur Zeit in Freiburg ein Projekt zur Erschließung der DMV-Akten von 1890 bis 1945 realisiert.

Meine Amtszeit als DMV-Präsidentin läuft in diesem Jahr aus, und ich freue mich sehr, daß Herr Hoffmann, der derzeitige DMV-Vizepräsident und ehemalige Vorsitzende des Wissenschaftsrates, sich bereit erklärt hat, für das Amt des Präsidenten 1998/99 zu kandidieren. Ich freue mich auch, daß Herr Janßen und Herr Wildenhain bereit sind, noch für eine zweite Amtsperiode im Präsidium mitzuarbeiten.
Das große Mathematikereignis, der ICM'98, rückt immer näher. Die ICM-Proceedings werden als Sonderband der DMV-Zeitschrift DocumentA MATHEMATICA herauskommen. Die Hauptvorträge werden nach Eingang frei elektronisch verfügbar sein und später in gedruckter Ausgabe an alle Teilnehmer und Teilnehmerinnen des ICM verschickt. Die DMVJahrestagung entfällt 1998 wegen des ICM, und anstelle der Studentenkonferenz wird es eine Satellitentagung „Junior Mathematical Congress“ vom 17. bis 22. August 1998 in Potsdam geben.

Die Vorbereitungen für die Jahrestagung vom 5. bis 12. September 1999 in Mainz haben begonnen, und das Programm-Komitee ist schon bei der Arbeit. Im Jahr 2000 findet die Jahrestagung in Dresden statt, und 2001 sind wir dann wieder wie in diesem Jahr bei der Österreichischen Mathematischen Gesellschaft zu Gast. Zusätzlich ist vom 7. bis 9. Juni 2001 ein Treffen der DMV mit der Belgischen Mathematischen Gesellschaft in Liège geplant. Auch hat das Präsidium schon über den Ort der Jahrestagung 2002 entschieden. Diese Tagung wird in Halle stattfinden.

Doch zunächst freuen wir uns auf die Studentenkonferenz in Halle am 4. und 5. September 1997 und auf die Jahrestagung in Salzburg vom 21. bis 26. September 1997.

Mit herzlichen Grüßen

Ihre Ina Kersten

Herzlich Willkommen:

\section{4. Österreichischer Mathematikerkongress}

\section{2. bis 26. September 1997 Universität Salzburg}

Für weitere und last minute Informationen:

* WWW-Homepage: http://random.math.sbg.ac.at/ peter/oemg97/

* Johannes Czermak — FAX: +43-662-8044-137

* Peter Zinterhof - e-mail: peter.zinterhof@sbg.ac.at 